\title{
Detection of Coulomb Charging around an Antidot in the Quantum Hall Regime
}

\author{
M. Kataoka, ${ }^{1}$ C. J. B. Ford, ${ }^{1}$ G. Faini,${ }^{2}$ D. Mailly,${ }^{2}$ M. Y. Simmons, ${ }^{1}$ D. R. Mace, ${ }^{1, *}$ C.-T. Liang, ${ }^{1}$ and \\ D. A. Ritchie ${ }^{1}$ \\ ${ }^{1}$ Cavendish Laboratory, Madingley Road, Cambridge CBз OHE, United Kingdom \\ ${ }^{2}$ Laboratoire de Microstructures et de Microelectronique - CNRS, 196, Avenue Henri Ravera, 92220 Bagneux, France
}

(August 6, 2018)

\begin{abstract}
We have detected oscillations of the charge around a potential hill (antidot) in a two-dimensional electron gas as a function of a large magnetic field $B$. The field confines electrons around the antidot in closed orbits, the areas of which are quantised through the Aharonov-Bohm effect. Increasing $B$ reduces each state's area, pushing electrons closer to the centre, until enough charge builds up for an electron to tunnel out. This is a new form of the Coulomb blockade seen in electrostatically confined dots. Addition and excitation spectra in DC bias confirm the Coulomb blockade of tunneling.
\end{abstract}

PACS numbers: 73.23.Hk, 73.40.Gk, 73.40.Hm

This paper addresses the fundamental question of whether charging can occur in an open system. Coulomb blockade $(\mathrm{CB})$ of tunnelling is generally only observed in electrostatically confined "dots" where there is only partial transmission through the entrance and exit constrictions. It has recently been seen when one constriction is open [1], when both constrictions transmit exactly one one-dimensional (1D) channel [2], or when some transmitted channels are decoupled from trapped states [3]. However, an unambiguous demonstration requires a completely open system, such as an antidot, which is a potential hill in a two-dimensional electron gas (2DEG). When a magnetic field $B$ is applied perpendicular to the 2DEG, a set of states, discrete in position and energy, is formed around the antidot, for each Landau level (LL). Aharonov-Bohm (AB) conductance oscillations arising from resonances through such states have been studied extensively in the integer and fractional quantum Hall $(\mathrm{QH})$ regimes [4] 9 . It has often been assumed that $\mathrm{CB}$ does not occur with antidot states because, as charge tries to build up, the system must immediately respond to screen it. However, pairs of $\mathrm{AB}$ oscillations from the two spins of the lowest LL were found to lock in antiphase, and this was attributed to charging [4,5]. In a dot system, it was suggested that the charging of edge channels is responsible for a similar regularity of the magnetoconductance peaks [10,11.

The aim of the present work was to detect such charge oscillations of an antidot, utilising a non-invasive voltage probe similar to that employed by Field et al. [12]. They fabricated a $1 \mathrm{D}$ constriction as a charge detector next to a dot but in a different circuit separated from it by a narrow gate. When the constriction was nearly pinched off, its resistance was very sensitive to potential variations nearby, and hence it could detect charge oscillations in the dot. We have fabricated a similar device with an antidot instead of a dot (see inset to Fig. 1(b)). A charging signal with the same period as the $\mathrm{AB}$ oscillations in the conductance $G_{\text {ad }}$ is clearly visible. The lineshape and phase show that $\mathrm{CB}$ of tunnelling through the antidot is occurring. DC-bias measurements are used to measure addition and excitation spectra, confirming this interpretation. The charging energy saturates at high $B$ and the single-particle (SP) energy spacing varies as $1 / B$. (a) $\nu_{\mathrm{c}}=\mathbf{2}$ (b) $\boldsymbol{\nu}_{\mathrm{c}}<1$

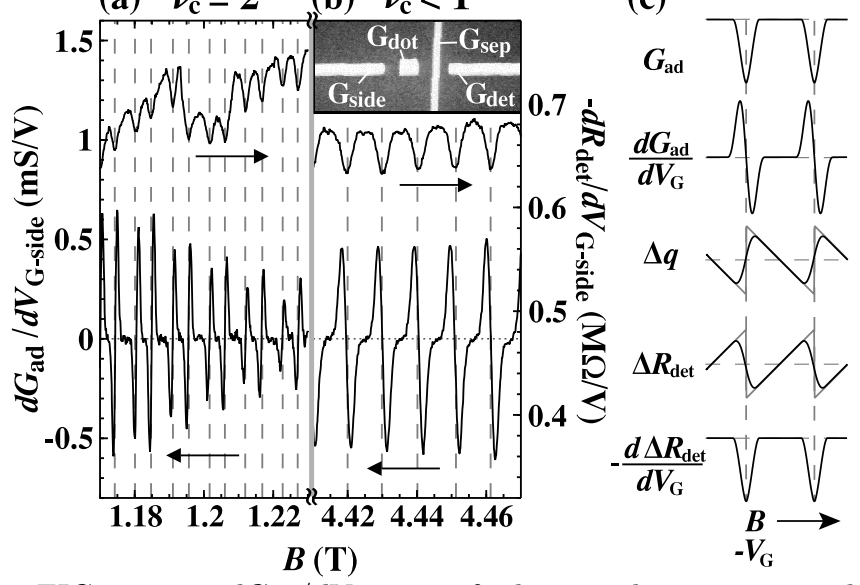

FIG. 1. $d G_{\mathrm{ad}} / d V_{\mathrm{G}-\text { side }}$ of the antidot circuit and $-d R_{\mathrm{det}} / d V_{\mathrm{G}-\text { side }}$ of the detector circuit with the gate voltage on $\mathrm{G}_{\text {side }}$ modulated in two different regimes: (a) $\nu_{\mathrm{c}}=2$ and (b) $\nu_{\mathrm{c}}<1$. Vertical dashed lines show the alignment of the dips in the detector signal with zeros in the transconductance oscillations. Inset: SEM micrograph of a device prior to second metallisation. (c) Illustration of the relation between various lineshapes. Grey lines in $\Delta q$ and $\Delta R_{\text {det }}$ are the ideal case, and black curves represent thermally broadened lineshapes.

The devices were fabricated from a GaAs/AlGaAs heterostructure with a 2DEG of sheet carrier density $2.2 \times 10^{15} \mathrm{~m}^{-2}$ and mobility $370 \mathrm{~m}^{2} / \mathrm{Vs}$ after illumination by a red LED. An SEM micrograph of a device is shown in the inset to Fig. 1 (b). A square dot gate $\left(\mathrm{G}_{\mathrm{dot}}\right), 0.3 \mu \mathrm{m}$ on a side, was contacted by a second metal layer evaporated on top of an insulator (not shown), to 
allow independent control of gate voltages. The lithographic widths of the antidot and detector constrictions were $0.45 \mu \mathrm{m}$ and $0.3 \mu \mathrm{m}$ respectively. All constrictions showed good $1 \mathrm{D}$ ballistic quantisation at $B=0$. A voltage of $-4.5 \mathrm{~V}$ on the separation gate $\left(\mathrm{G}_{\mathrm{sep}}\right)$, of width $0.1 \mu \mathrm{m}$, divided the 2DEG into separate antidot and detector circuits. The detector gate $\left(\mathrm{G}_{\mathrm{det}}\right)$ squeezed the detector constriction to a resistance between 0.1 and $5 \mathrm{M} \Omega$ to make it very sensitive to nearby charge. To maximise the sensitivity transresistance measurements were made by modulating the dot-gate voltage (or the voltage on the side-gate $\mathrm{G}_{\text {side }}$ ) at $10 \mathrm{~Hz}$ with $0.5 \mathrm{mV}$ rms and applying a DC current of $1 \mathrm{nA}$ through the detector constriction. Simultaneously, the transconductance of the antidot circuit was measured with a $10 \mu \mathrm{V}$ DC source-drain bias, when necessary. The experiments were performed in a dilution refrigerator with a base temperature below $50 \mathrm{mK}$.

Figure 11 shows the transresistance $-d R_{\text {det }} / d V_{\mathrm{G}-\text { side }}$ (transconductance $\left.d G_{\mathrm{ad}} / d V_{\mathrm{G}-\text { side }}\right)$ vs $B$ of the detector (antidot) circuit in two different field regions: (a) $\nu_{\mathrm{c}}=2$ and (b) $\nu_{\mathrm{c}}<1$, where $\nu_{\mathrm{c}}$ is the filling factor in both antidot constrictions, which were determined from $G_{\text {ad }}$. The filling factors in the bulk 2DEG were $\nu_{\mathrm{b}}=7$ and 2 , respectively. The oscillations in $G_{\mathrm{ad}}$ occur as SP states around the antidot rise up through the Fermi energy $E_{\mathrm{F}}$. The AB effect causes the overall pe$\operatorname{riod} \Delta B$ to be $h / e S$, where $S$ is the area enclosed by the state at $E_{\mathrm{F}}$. The curve in (a) has pairs of spin-split peaks, whereas in (b) only one spin of the lowest LL is present. The dips in $-d R_{\text {det }} / d V_{\mathrm{G}-\text { side }}$ correspond to a saw-tooth in the change $\Delta R_{\text {det }}$ from the background resistance (see Fig. 1 $1(\mathrm{c})$ ). Thus the net charge $\Delta q$ nearby suddenly becomes more positive (making the effective gate voltage less negative) whenever the antidot comes on to resonance (since the dips line up with the zeros in $\left.d G_{\mathrm{ad}} / d V_{\mathrm{G}-\text { side }}\right)$. Hence we conclude that this charge oscillation is associated with states near the antidot. A second sample showed very similar results.

We explain the charging as follows. As $B$ increases, all the states encircling the antidot move inwards, reducing their areas to keep the flux enclosed constant, and hence a net charge $\Delta q$ builds up in the region around the antidot. This resembles CB in a dot [13]. At low bias, the electron in the highest occupied state cannot escape until $\Delta q$ reaches $-e / 2$, then it tunnels out to a nearby lead or into a localised state in the bulk, and $\Delta q$ becomes $+e / 2$. At this point charge can move easily through the antidot, and so each dip in the detector signal lines up with such a conductance resonance, as found experimentally (Fig. 1). There is no electrostatically confined region around the antidot, so charging seems impossible [6]. However, electrons are magnetically confined to the antidot and the rigidity of the quantum-mechanical orbitals prevents charge relaxation. Other states further away from the antidot might try to screen the charge build-up. However, those in the same LL have a fixed density once it is full, and so cannot screen. Also, due to the discreteness of the SP states, rearrangement of charge below $E_{\mathrm{F}}$ within the partially filled region near the antidot can only cause discrete changes in the charge, and would probably cost too much interaction energy. One might speculate that the detector would pick up not the charging of the antidot but the change in screening by SP states near $E_{\mathrm{F}}$ because they could adjust their areas or the wavefunction could leak out to the other edges on resonance [14]. However, such screening should be symmetric around the resonances. Therefore the transresistance would be the derivative of periodic dips or peaks, not of a saw-tooth as seen in our measurements.

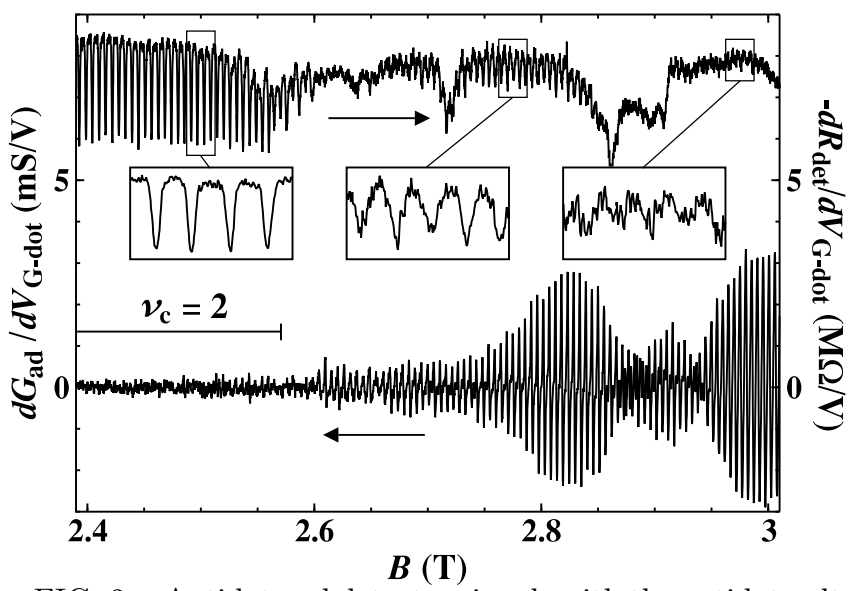

FIG. 2. Antidot and detector signals with the antidot voltage modulated, in the regime of pure $h / 2 e \mathrm{AB}$ oscillations. The amplitude of the detector oscillations (upper curve) suddenly decreases at the onset of the oscillations in the antidot circuit (lower curve).

The charging of the antidot is not dependent on the presence of conductance oscillations in the antidot circuit. Thus it is still possible to observe the signal with no applied bias in the antidot circuit, or when the sidegate voltage is zero so that there is no tunnelling between that edge and the antidot. Indeed, as shown in Fig. 2, the dips in the detector signal become large and sharp when the antidot constrictions are set to a $\mathrm{QH}$ plateau $\left(\nu_{\mathrm{c}}=2\right.$ in this case), where the antidot states are decoupled from the extended edge states. Away from the QH plateau, since the states are coupled to the current leads, electrons' wavefunctions penetrate into the leads, reducing the effective maximum charge on the antidot and leading to weaker charging, i.e. smaller charging energy.

Around $B=3 \mathrm{~T}$ the spin-splitting of the peaks becomes exactly half the period, and the amplitudes of the two peaks in each pair become identical, giving what appear to be $h / 2 e \mathrm{AB}$ oscillations (see Fig. 2) [4, 5]. We have investigated the temperature dependence of both the charging and conductance signals in this regime. The Fourier transforms of the charging signal appearing at 
around 2.5 T in Fig. 2 and the $G_{\text {ad }}$ oscillations at around $2.8 \mathrm{~T}$, measured separately, decrease at different rates (see Fig. 3(a)). Thermally broadened Fermi-liquid theory for sinusoidal oscillations [8] gives a good fit for $G_{\text {ad }}$ with an energy level spacing of $70 \mu \mathrm{eV}$. The conductance oscillations are suppressed at high temperature because of thermal broadening of the edge channels around the side gates at $E_{\mathrm{F}}$ when the thermal energy becomes comparable to the sum of the SP energy spacing and the charging energy $e^{2} / C$ (if CB occurs), where $C$ is the total capacitance of the antidot. For the charging signal, since the oscillations are not sinusoidal, a more detailed model [13] is required than that used above. Here, we assume that the detector is only sensitive to thermal excitation which adds or removes electrons around the antidot, but not to excitation between SP states.

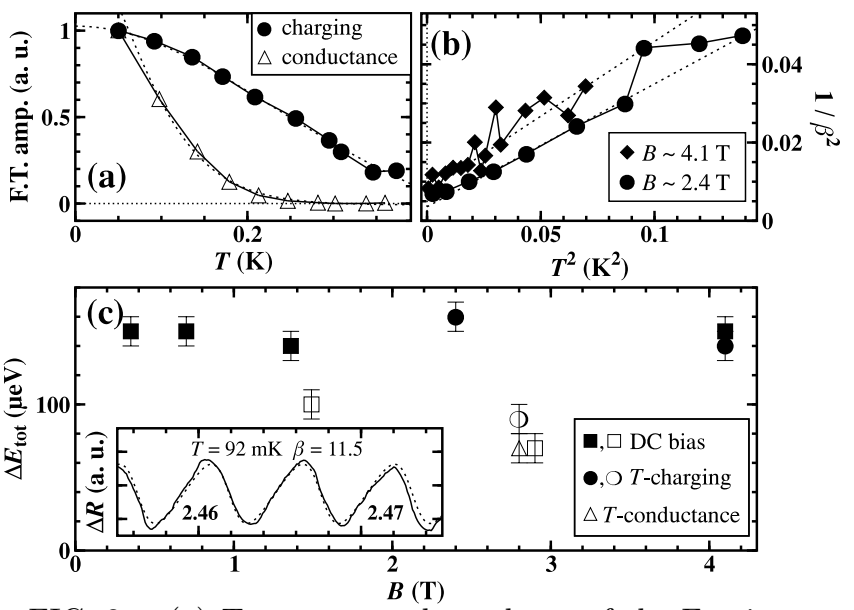

FIG. 3. (a) Temperature dependence of the Fourier amplitude of $h / 2 e$ oscillations in conductance (not transconductance) (triangles, $B \approx 2.8 \mathrm{~T}$ ) and charging (circles, $B \approx 2.5 \mathrm{~T}$ ). The amplitudes were calculated by taking the square root of the Fourier power spectrum integrated around the $h / 2 e$ peak. Dashed lines are fits to thermally broadened Fermi-liquid theory. (b) $\beta$ (see text) vs $T^{2}$ for $h / 2 e$ (circles) and $h / e$ (diamonds) $\mathrm{AB}$ oscillations. The straight lines are fits. (c) $\Delta E_{\text {tot }}$ obtained from the measurements shown. Filled (open) symbols correspond to $G_{\text {ad }}$ on (below) the $\nu_{\mathrm{c}}=2$ plateau, except for the highest $B$ data which is on the $\nu_{\mathrm{c}}=1$ plateau. Inset: integral of the detector oscillations. The dashed line is a fit as described in the text.

The electrochemical potential of the antidot $\mu_{\mathrm{ad}}(N, B)$ is the energy required to add an electron to the lowest unoccupied state, which encloses, say, $N$ flux quanta $h / e$. Then the probability that thermal excitation moves an electron from a lead at chemical potential $\mu$ to that state is given by the Fermi function $f\left(\mu_{\mathrm{ad}}(N, B)-\mu\right)$. For one period $-\frac{\Delta B}{2}<B<\frac{\Delta B}{2}$, where the centre of the charge transition is at $B=0$, the blurred sawtooth charge oscillation can be written as $\Delta q(B)=$ $-e\left(B / \Delta B+f\left(\mu_{\mathrm{ad}}(N, B)-\mu\right)-\frac{1}{2}\right)$. Since the charging energy is parabolic in the net charge, and hence varies as $\left(B \pm \frac{\Delta B}{2}\right)^{2}$ depending on which state is occupied, it can be shown simply that $\mu_{\text {ad }}(N, B)-\mu=\Delta E_{\text {tot }} B / \Delta B$, where $\Delta E_{\text {tot }}=\Delta E+e^{2} / C$. Here, $\Delta E$ is the average energy spacing of adjacent states (of whichever spin), equal to $\Delta E_{\mathrm{sp}} / 2$ when both spins encircle the antidot; $\Delta E_{\mathrm{sp}}$ is the energy spacing of adjacent SP states of the same spin. For $h / 2 e$ oscillations, we assume that a spindown state lies midway in energy between spin-up states. For $\nu_{\mathrm{c}} \leq 1, \Delta E=\Delta E_{\mathrm{sp}}$. Thus $f\left(\mu_{\mathrm{ad}}(N, B)-\mu\right)=$ $(1+\exp (-\beta B / \Delta B))^{-1}$ where $\beta=\Delta E_{\text {tot }} / k_{\mathrm{B}} T^{*}$. Here $T^{*}=\sqrt{T^{2}+\Gamma^{2}}$ is the effective temperature, to account for an intrinsic broadening $\Gamma$ at low temperatures due to the $\mathrm{AC}$ excitation voltage and the finite lifetime of the states. The integral of the detector signal with respect to $B$ (approximately equivalent to the integral with respect to $\left.-V_{\mathrm{G}-\text { dot }}\right)$ was fitted to $\Delta q(B)$, after subtracting the background slope (inset to Fig. $3(\mathrm{c})$ ). From the fit at various temperatures (Fig. 3(b), circles), we obtained $\Delta E_{\mathrm{tot}}=160 \mu \mathrm{eV}$. We could not measure the temperature dependence in the region $B \approx 2.8 \mathrm{~T}$ in Fig. 2 due to the small charging signal. However, a fit to the data at $T \approx 50 \mathrm{mK}$ gave $\Delta E_{\text {tot }}=90 \mu \mathrm{eV}$, assuming that $\Gamma$ does not change. The temperature dependence of $h / e$ oscillations where $\nu_{\mathrm{c}}$ was just less than one $(B \approx 4.1 \mathrm{~T}$, diamonds in Fig. 3(b)) gave $\Delta E_{\text {tot }}=140 \mu \mathrm{eV}$. These energies are plotted in Fig. 3(c) and will be discussed below.

A further way of measuring the energy spacing is to apply a DC bias 15. Fig. A shows greyscale plots of the DC-bias dependence of $\mathrm{AB}$ oscillations in the differential conductance (measured with a $5 \mu \mathrm{V}$ rms $\mathrm{AC}$ $(10 \mathrm{~Hz})$ source-drain voltage in addition to the DC bias), for the values of $\nu_{\mathrm{c}}$ shown. In (a) and (b), peaks are shown in black, since resonant transmission occurs due to inter-LL scattering [7]. This is not present at higher $B$; instead, resonant backscattering gives dips (shown in black in (c) and (d)). (a)-(c) show sets of spin-split resonances. In (a), where spin splitting is poorly resolved, adjacent peaks cross at $250 \mu \mathrm{V}$ or $50 \mu \mathrm{V}$. As energies, since this is an addition spectrum, these correspond to $e^{2} / C+\Delta E_{\mathrm{sp}}-E_{\mathrm{Z}}$ and $e^{2} / C+E_{\mathrm{Z}}$ respectively, where $E_{\mathrm{Z}}$ is the Zeeman splitting. Thus the average energy is just $\Delta E_{\text {tot }}$. This enables a comparison of energies at various $B$ (see Fig. 3(c)). At higher $B$, spin-splitting becomes obvious (Figs. $4(\mathrm{~b})$ and (c)), but the crossings give similar $\Delta E_{\text {tot }}$.

The DC bias at which states of different spin cross gives an upper limit for $e^{2} / C$, and this limit increases with $B$, as does $E_{\mathrm{Z}}$. It is likely that the charging energy is small at low $B$, since the magnetic confinement is weak; indeed, the charging signal is hard to see for $B<0.6 \mathrm{~T}$. However, at $B=1.4 \mathrm{~T}$, in the middle of Fig. A(c), $\Delta E_{\text {tot }}$ drops rapidly by $30 \%$ (open symbol in Fig. $3(\mathrm{c}))$. This corresponds to the field at which the conductance falls off the $\nu_{\mathrm{c}}=2$ plateau (for these particular gate voltages). The figure shows a similar drop (for 


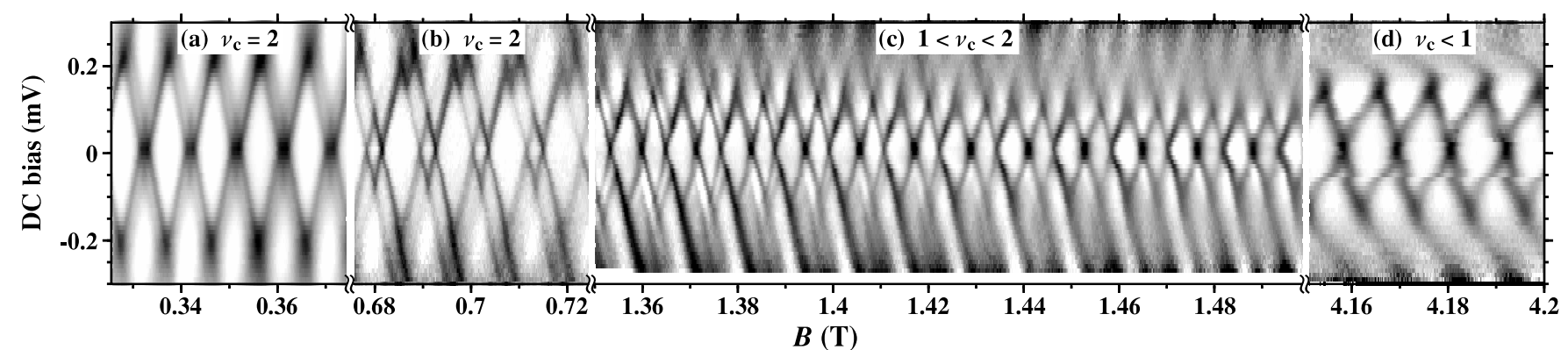

FIG. 4. Greyscale plots of DC-bias dependence of the differential conductance of the antidot at various $B$. The same gate voltages were used throughout. Dark regions correspond to positions of peaks or dips. The background variation in the signal was subtracted to increase the contrast.

the gate voltages used in the temperature dependence described above), around $2.6 \mathrm{~T}$, also corresponding to moving off the $\nu_{\mathrm{c}}=2$ plateau. Temperature dependences of the conductance and charging oscillations there confirm the DC bias result. There is no reason why $\Delta E_{\mathrm{sp}}$ should change so suddenly. These drops occur when the coupling of the antidot to the leads increases, reducing the charging energy, as described above.

In Fig. 国(c) additional parallel lines appear around the smaller diamonds, offset by $60 \mu \mathrm{V}$ in DC bias. We interpret these as arising from tunnelling via the first excited state of the antidot, which is $\Delta E_{\mathrm{sp}}-E_{\mathrm{Z}}$ higher in energy. Similar lines are not resolved around the larger diamonds since the spacing is just $E_{\mathrm{Z}}$. The observation of this excitation spectrum confirms that there is a Coulomb blockade of tunnelling through the antidot.

For a constant potential slope, $\Delta E_{\mathrm{sp}}$ should vary as $1 / B$. At $B=0.35 \mathrm{~T}, \Delta E_{\text {tot }}=150 \mu \mathrm{eV}$ and $e^{2} / C<50 \mu \mathrm{eV}$ (the upper limit from the DC-bias measurements), so $200 \mu \mathrm{eV}<\Delta E_{\mathrm{sp}}<300 \mu \mathrm{eV}$. Thus at $B=1.4 \mathrm{~T}$ we expect $50 \mu \mathrm{eV}<\Delta E_{\mathrm{sp}}<80 \mu \mathrm{eV}$. This is close to the value $\Delta E_{\mathrm{sp}} \approx 100 \mu \mathrm{eV}$ obtained from the addition and excitation spectra at $1.4 \mathrm{~T}$, which also give $E_{\mathrm{Z}} \approx 35 \mu \mathrm{eV}$, in good agreement with $g \mu_{\mathrm{B}} B$ with $g=0.44$ for electrons in GaAs. From Fig. 4 (c), $e^{2} / C=\Delta E_{\mathrm{tot}}-\Delta E_{\mathrm{sp}} / 2$ falls from $\approx 100 \mu \mathrm{eV}$ on the plateau to $\approx 65 \mu \mathrm{eV}$ when the antidot is coupled to the leads. When on the plateau, $e^{2} / C$ appears to saturate at $\approx 150 \mu \mathrm{eV}$ above $B \approx 2 \mathrm{~T}$, since by then the states around the antidot are well defined and so the full $\pm e / 2$ charge can build up, with the capacitance fairly constant. Maasilta and Goldman [9] found from the lineshapes of individual peaks at $\nu=1$ and $\frac{1}{3}$ that $\Delta E_{\text {tot }}$ was almost constant, but interpreted this as a self-consistent variation of the potential slope, with no CB. In our picture, the constancy of $\Delta E_{\mathrm{tot}}$ comes from the interplay of $e^{2} / C$ and $\Delta E_{\mathrm{sp}}$.

In summary, we have fabricated a charge detector in close proximity to an antidot. The antidot is seen to discharge each time a state around the antidot comes on to resonance, showing that there is a Coulomb blockade of tunnelling via the antidot. We have measured addition and excitation spectra, confirming this interpretation. The charging energy drops whenever there is coupling to the leads, as the charge becomes delocalised. This is the first conclusive demonstration of charging in an open system. It arises from the rigidity of the quantummechanical wavefunction, as for an electron in an atom. It must form part of the explanation for the pure $h / 2 e$ $\mathrm{AB}$ oscillations 沟.

This work was funded by the UK EPSRC. We thank C. H. W. Barnes and C. G. Smith for useful discussions. M. K. acknowledges financial support from Cambridge Overseas Trust.

*Present address: The Technology Partnership PLC, Melbourn Science Park, Melbourn, SG8 6EE, UK.

\section{References}

[1] C. Pasquier et al., Phys. Rev. Lett. 70, 69 (1993); C. H. Crouch et al., Superlatt. and Microstr. 20, 377 (1996).

[2] S. M. Cronenwett et al., Phys. Rev. Lett. 81, 5904 (1998).

[3] C. T. Liang et al., Phys. Rev. Lett. 81, 3507 (1998).

[4] C. J. B. Ford et al., Phys. Rev. B 49, 17456 (1994).

[5] A. S. Sachrajda et al., Phys. Rev. B 50, 10856 (1994).

[6] V. J. Goldman and B. Su, Science 267, 1010 (1995).

[7] D. R. Mace et al., Phys. Rev. B 52, R8672 (1995).

[8] J. D. F. Franklin et al., Surf. Sci. 361/362, 17 (1996).

[9] I. J. Maasilta and V. J. Goldman, Phys. Rev. B 57, R4273 (1998).

[10] M. W. C. Dharma-Wardana, R. P. Taylor, and A. S. Sachrajda, Solid State Commun. 84, 631 (1992).

[11] A. Sachrajda et al., Surf. Sci. 305, 527 (1994).

[12] M. Field et al., Phys. Rev. Lett. 70, 1311 (1993).

[13] C. W. J. Beenakker, Phys. Rev. B 44, 1646 (1991).

[14] M. Field et al., Phys. Rev. Lett. 77, 350 (1996).

[15] E. B. Foxman et al., Phys. Rev. B 47, 10020 (1993). 